up. In the non-PPM group, 3 patients had a RBBB pattern and 15 had a LBBB pattern at baseline. Patients who had a baseline RBBB were more likely to require a PPM post TAVI (p-value $=0.02)$.

Conclusion Baseline RBBB ECG pre-TAVR is a significant predictor of delayed advanced AV block. Our study validates previous suggestions that patients with baseline RBBB should not be considered for early discharge and may require prolonged monitoring to detect delayed AV conduction abnormalities. These patients are less likely to recover from their conduction disturbances as suggested by their pacing requirement at 6 week post-implantation follow-up.

\section{IMPACT OF THE 2019 ESC CHRONIC CORONARY SYNDROME GUIDELINES PUBLICATION, ON REFERRALS FOR CORONARY IMAGING IN A CHEST PAIN SERVICE}

M Waters, C O' Conor, G Offiah, S Ingram, B Loo. Tallaght University Hospital, Dublin, Ireland

10.1136/heartjnl-2020-ICS.13

On August 31st 2019 the ESC guidelines for the diagnosis and management of chronic coronary artery syndrome was published. The guidelines give a greater emphasis on the use CT coronary arteries (CTCA) in the work-up of obstructive coronary artery disease (CAD). In addition, the use of exercise stress testing (EST) in establishing a diagnosis of coronary artery disease was downgraded from Class I recommendation to Class IIb. The guidelines also reduced the pre-test probability (PTP) of obstructive coronary artery disease in symptomatic patients according to age, sex and nature of symptoms. However, currently the additive effects of risk factors and EST is unknown. The aim of this study was to assess whether publication of the ESC 2019 guidelines had an effect on referrals for further coronary imaging in patients that were referred to the chest pain service, without a history of coronary artery disease.

Methods A retrospective review of patients referred to the chest pain service in 2019. Only patients without a prior history of coronary artery disease, with negative troponins and without dynamic ECG changes were included in our analysis. All patients underwent exercise stress testing at baseline. A comparison of referrals for invasive coronary angiogram or CTCA, between the periods of January 1st 2019 - 31st of August, with September 1st - 31st December 2019 post publication of the ESC guidelines was made. PTP was calculated via the 2019 ESC guidelines.

Results The analysis includes 330 patients referred to the chest pain services without a prior history of coronary artery disease. Baseline characteristics; 49.39\% male, mean age 55.3 yrs, negative EST $68.8 \%$, baseline PTP 10\%. Further coronary imaging was selected in $45.7 \%(\mathrm{n}=151)$ of patients seen by the chest pain service in 2019, mean PTP 12\%.

Prior to publication of the ESC guidelines, further coronary imaging was performed in $41.30 \%$ of patients seen by the chest pain services; compared to $57.2 \%$ after the publication of the ESC guidelines, p-value 0.02. The increase in imaging referrals reflect the increased use of CTCA post guideline publication, $16.4 \%$ vs $29.5 \%$, p-value 0.003 . CTCA was performed mainly in patients with low PTP mean 7.5\%, nonanginal chest pain $83.8 \%(\mathrm{n}=57)$ and negative EST $92.6 \%$ $(n=63)$.
The incidence of obstructive CAD in our cohort was 9.2\% ( $\mathrm{n}=14$ ); mean PTP 18\%, 84.6\% positive EST. The negative predictive value of a negative or sub-maximal EST was $98.13 \%$ in our cohort who had further imaging $(95 \% \mathrm{CI}$ 92.8 - 99.4\%).

Discussion The publication of the ESC 2019 guidelines on chronic coronary syndromes, coincided with an increase in CTCA referrals by $180 \%$ in the chest pain service. The overall incidence of obstructive coronary artery disease was low, which is likely reflected by the low PTP in this cohort. Our results underline the utility of EST in risk assessing the probability of obstructive CAD in this cohort, however physicians need to understand its diminished role in the diagnosis of coronary artery disease.

\section{ANTITHROMBOTIC THERAPY WITH OR WITHOUT ASPIRIN AFTER PERCUTANEOUS CORONARY INTERVENTION OR ACUTE CORONARY SYNDROME IN PATIENTS TAKING ORAL ANTICOAGULATION: A META- ANALYSIS AND NETWORK ANALYSIS OF RANDOMISED CONTROLLED TRIALS}

${ }^{1,2} \mathrm{R}$ Colleran, ${ }^{1,3} \mathrm{RA}$ Byrne, ${ }^{1} \mathrm{H}$ Rai, ${ }^{2} \mathrm{~A}$ Kastrati, ${ }^{2} \mathrm{~S}$ Cassese. ${ }^{1}$ Cardiovascular Research Institute Dublin, Mater Private Hospital, Dublin, Ireland; ${ }^{2}$ German Heart Centre, Technical University of Munich, Munich, Germany; ${ }^{3}$ School of Pharmacy and Biomolecular Sciences, Royal College of Surgeons University of Medicine and Health Sciences, Dublin, Ireland

\subsection{6/heartjnl-2020-ICS.14}

Introduction Trials investigating aspirin-omission in patients taking oral anticoagulation (OAC) after percutaneous coronary intervention (PCI) or acute coronary syndrome (ACS) were not powered to assess rates of major bleeding or ischaemic events.

Methods We performed an updated meta-analysis and network analysis of randomised trials comparing treatment with or without aspirin in patients taking OAC and a P2Y12-inhibitor after PCI or ACS. The primary outcome was TIMI major bleeding.

Results Five trials enrolling 11,542 patients allocated to antithrombotic regimens omitting $(n=5,795)$ or including aspirin $(n=5,747)$ were included. Aspirin-omission was associated with a lower risk of TIMI major bleeding $(R R=0.56$, 95\% CI [0.44-0.71]; $\mathrm{P}<0.001)$ but a trend towards a higher risk of $\mathrm{MI}(\mathrm{RR}=1.21,95 \% \mathrm{CI}$ [0.99-1.47]; $\mathrm{P}=0.06)$, which was significantly higher when only non-vitamin $\mathrm{K}$ antagonist OAC (NOAC)-based trials were considered ( $\mathrm{P}_{\text {interac- }}$ tion $=0.02$ ). The risk of stent thrombosis was comparable with both strategies $(\mathrm{RR}=1.29$, 95\%CI [0.87-1.90]; $\mathrm{P}=$ 0.20 ), with a trend towards a higher risk of ST with aspirin-omission when only NOAC-based trials were considered $\left(P_{\text {interaction }}=0.06\right)$. Risks of stroke and death were similar with both strategies. Network meta-analysis ranked dabigatran (low dose) without aspirin as the best strategy for bleeding reduction $(\mathrm{P}$-score $=0.86)$ and apixaban with aspirin as the best strategy for MI reduction (P-score= 0.66). (Figure 1).

Conclusions In patients taking OAC after PCI or ACS, aspirinomission is associated with a lower risk of TIMI major bleeding, with a possible increased risk of thrombotic events, which is more marked when only NOAC-based trials are considered. This supports individualization of the treatment regimen based on patient risk. 